\title{
High-Level Magnetic Activity on a Low-Mass Close Binary: GSC 02038-0293
}

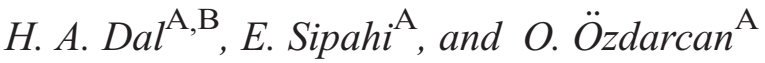 \\ A Department of Astronomy and Space Sciences, University of Ege, Bornova, 35100 İzmir, Turkey \\ ${ }^{\mathrm{B}}$ Corresponding author. Email: ali.dal@ege.edu.tr
}

\begin{abstract}
Taking into account results obtained from light-curve analysis and out-of-eclipse analyses, we discuss the nature of GSC 02038-00293 and also its magnetic activity behaviour. We obtained light curves of the system during observing seasons 2007, 2008 and 2011. We obtained its secondary minimum clearly in $I$-band observations in 2008 for the first time. Analysing this light curve, we found the physical parameters of the components. The light-curve analysis indicates that the possible mass ratio of the system is 0.35 . We obtained the remaining $V$-band light curves, extracting the eclipses. We modelled these remaining curves using the SPOTMODEL program and found possible spot configurations of the magnetically active component for each observing season. The models demonstrated that there are two active longitudes for the active component. The models reveal that both active longitudes migrate in the direction of decreasing longitude. We also examined the light curves in out-of-eclipse phases with respect to minimum and maximum brightness, amplitude, etc. The amplitude of the curves during out-of-eclipse phases varies in a sinusoidal way with a period of $\sim 8.9 \mathrm{yr}$; the mean brightness of the system is dramatically decreasing. The phases of the deeper minimum during out-of-eclipse periods exhibit a migration toward decreasing phase.
\end{abstract}

Keywords: stars: activity — binaries: eclipsing — stars: individual: GSC 02038-0293 — stars: low-mass — starspots

Received 2012 January 16, accepted 2012 February 24, published online 2012 March 27

\section{Introduction}

Many stars, such as BY Dra and RS CVn type stars on the main sequence toward late spectral types, exhibit stellar spot activity referred to as magnetic activity. In the literature, BY Dra variables were found for the first time by Kron (1952), who demonstrated some sinusoidal-like variations at out-of-eclipse stages, hereafter 'out-ofeclipses', of the eclipsing binary star YY Gem. He explained the variation during out-of-eclipses as the result of heterogeneous temperature at the stellar surface. Such stars were then named 'BY Dra stars' by Kunkel (1975). Based on some rigorous arguments obtained from observations, the variability seen in BY Dra type stars was confirmed in terms of dark regions on the surface of the stars in later works such as those of Bopp \& Evans (1973), Torres \& Ferraz Mello (1973), Friedemann \& Gürtler (1975), Vogt (1975), and Bopp \& Fekel (1977). RS Cvn type stars exhibit the same variability as BY Dra stars. However, one of the components of a RS CVn star is evolved and is generally a giant or subgiant star, while the other component is a main sequence star (Thomas \& Weiss 2008). The incidence of late-type stars in our Galaxy is about $65 \%$. $75 \%$ of late-type stars show magnetic activity such as spot and flare activity (Rodonó 1986). Research such as that of López-Morales (2007) and Morales et al. $(2008,2010)$ demonstrates that magnetic activity dramatically affects the stellar structure of late-type stars and also their evolution. Casagrande et al. (2008), Morales et al. (2008, 2010), Fernandez et al. (2009), Torres et al. (2010), and Kraus et al. (2011) have revealed that the radii found from analyses of observations are generally larger than the radii expected theoretically for several magnetically active low-mass binaries, while the effective temperatures found from observations are usually lower than those expected theoretically. They claim that the reason is most likely magnetic activity. In this respect, magnetically active low-mass components of binaries take a very important place in our understanding of stellar evolution.

In this study, we introduce GSC 02038-00293 as a new candidate for the class of magnetically active low-mass stars. Using the Robotic Optical Transient Search Experiment (ROTSE) 1 database (Woźniak et al. 2004), the system was discovered by Bernhard \& Frank (2006) in the optical identification programme of X-ray sources listed in the ROSAT All-Sky Survey Bright Source Catalogue (Voges et al. 1999). The identification reveals that the system is actually the uncatalogued variable Northern Sky Variability Survey (NSVS) object ID 7869362 as the optical counterpart of the X-ray source 1RXS J160248.3+252031. Combining their 2005 and 2006 data with the available data taken from the ROTSE 1 and All 
Sky Automated Survey (ASAS) 3 databases, Bernhard \& Frank (2006) determined light elements as follows: JD (Hel. $)_{\text {Min } I}=53560.491(3)+0^{\mathrm{d}}$. 49541(1) E. Norton et al. (2007) gave the same period after analysing Wide Angle Search for Planets (SuperWASP) observations of the system. In addition, Frank \& Bernhard (2007) also confirmed the period with 2007 observations. Bernhard \& Frank (2006) indicted a 6-8 yr activity cycle. However, consecutive observations demonstrated that the lightcurve shape can change even within one week. Finally, Bernhard \& Frank (2006) identified the system as a RS CVn type binary. Using low-resolution spectra, Dragomir et al. (2007) indicated that the spectral type of the system was likely to be K-type. A detailed spectral study was performed by Korhonen et al. (2010). Considering some features seen in the low-resolution spectra of the system, such as neutral metals $(\mathrm{Mg}, \mathrm{Na})$, weak Balmer lines, and also the absence of molecular bands, they confirmed that the system is from spectral type $\mathrm{K}$. Adopting $\log g=4.5$, $T_{\text {eff }}$ was found to be $4750 \pm 250 \mathrm{~K}$ and $v \sin i$ was found to be $90 \pm 10 \mathrm{~km} \mathrm{~s}^{-1}$. However, using their $v \sin i$ measured together with the rotation period of $0.495410 \mathrm{~d}$ found by Bernhard \& Frank (2006), Korhonen et al. (2010) estimated the system's radius as $R \sin i=0.88 \pm 0.10 \mathrm{R} \odot$. The value of $R \sin i$ indicated a spectral type of late $\mathrm{G}$ or later.

The observations in the literature have demonstrated that GSC 02038-00293 exhibits magnetic activity. In this study we analyse the light curves of the system, and also examine the variations during out-of-eclipses. We finally compare the system with its analogue with respect to theoretical models.

\section{Observations and Data}

Observations of GSC 02038-00293 were carried out with two telescopes in $B V R I$ bands at Ege University Observatory. The first group of observations was acquired with a High-Speed Three Channel Photometer attached to the 48-cm Cassegrain type telescope during observing seasons 2007 and 2008. The second group of observations was acquired with a thermoelectrically cooled ALTA $\mathrm{U}+422048 \times 2048$ pixel CCD camera attached to a 40-cm Schmidt-Cassegrain-type Meade telescope during the observing seasons 2008 and 2011. The comparison and check stars used in all observations are the same stars used in the literature. Some basic parameters of the programme stars are listed in Table 1. The names of the stars are listed in the first column, while the $\mathrm{J} 2000$ coordinates are listed in the second column. The $V$ magnitudes are in the third column and the $B-V$ colours are listed in the last column.

Although the programme and comparison stars are very close in the sky, differential atmospheric extinction corrections were applied. The atmospheric extinction coefficients were obtained from observations of the comparison stars on each night. Moreover, the comparison stars were observed with the standard stars in their vicinity and reduced differential magnitudes, in the sense variable minus comparison, were transformed to the standard system using the procedures outlined by Hardie (1962). The standard stars are listed in the catalogues of Landolt $(1983,1992)$. Furthermore, the dereddened colours of the system were computed. Heliocentric corrections were also applied to the times of the observations.

In $B V R$ bands, the first group of observations was continued for 6 nights between 2007 April 22 and July 19, and was carried on for 4 nights between 2008 April 7 and July 23 with the $48-\mathrm{cm}$ Cassegrain-type telescope. In addition, the second group of observations was continued for 9 nights between 2008 May 20 and August 20 in BVRI bands, and carried on for 2 nights in 2001, April 23 and May 9, with the 40-cm Schmidt-Cassegrain-type Meade telescope. The mean averages of the standard deviations were found to be $0.009,0.007$ and $0.007 \mathrm{mag}$ from the observations with the 48-cm Cassegrain-type telescope for the $B V R$ bands, respectively, and $0.023,0.011,0.010$ and 0.013 mag for observations acquired with the $40-\mathrm{cm}$ Schmidt-Cassegrain-type Meade telescope for the BVRI bands, respectively. To compute the standard deviations of observations, we used the standard deviations of the reduced differential magnitudes in the sense comparison minus check stars for each night. There was no variation observed in the standard brightness of comparison stars.

The times of minima obtained in this study are listed in Table 2. In the table, the first 9 minimum times have already been published by Sipahi et al. (2009), while the last one is unpublished. Using all available times of minima in the literature, we adjusted the light elements of the system, as follows:

$$
\mathrm{JD}(\text { Hel. })=2453560.4925(9)+0^{\mathrm{d}} .4954115(5) E .
$$

Using the light elements given by equation (1), we phased all our observations and also all data taken from the literature. In Figure 1, the light and colour curves of the system are shown for three observing seasons: 2007 ,

Table 1. Basic parameters for the observed stars

\begin{tabular}{|c|c|c|c|}
\hline $\begin{array}{l}\text { Star } \\
\text { name }\end{array}$ & $\begin{array}{c}\text { RA/Dec. (J2000) } \\
\left({ }^{\mathrm{h} \mathrm{ms}}\right) /\left({ }^{\circ}{ }^{\prime} \prime \prime\right)\end{array}$ & $\begin{array}{c}V^{a} \\
(\mathrm{mag})\end{array}$ & $\begin{array}{l}B-V \\
\text { (mag) }\end{array}$ \\
\hline GSC 02038-00293 & $160248.54+252038.9$ & 10.540 & 0.972 \\
\hline GSC 02038-00867 (Comparison) & $160535.85+251659.6$ & 9.185 & 1.243 \\
\hline GSC 02038-00565 (Check) & $160253.98+251043.2$ & 11.811 & 0.394 \\
\hline GSC 02038-00663 (Check) & $160313.37+251210.9$ & 11.513 & 0.738 \\
\hline
\end{tabular}

${ }^{a}$ The $V$-band brightness and $B-V$ index were obtained in this study. 
2008 and 2011. As can be seen from the figures, there is a remarkable variation in the shape of the light curves from one season to the next. As is well known from the literature, the system exhibits magnetic activity. Figure 1 demonstrates that the available magnetic activity causes dramatic distortion in the light-curve shape. Although the secondary minimum shows itself in observing season 2008 , it cannot clearly reveal itself in general. In addition, the $B-V$ colour curves exhibit some variations, while there is no clear variation over the standard deviation in the $V-R$ colour curves. However, we had a chance to observe the system in the $I$ band during the programme of the 40-cm Schmidt-Cassegrain-type Meade telescope for both seasons 2008 and 2011. I-band observations show the secondary minimum better than all other bands (see Section 3). The secondary minimum exhibits itself better toward long wavelengths.

For analysis purposes, we collected all available data from the literature. With this aim in mind, we obtained the

Table 2. Times of minima of GSC 02038-00293

\begin{tabular}{lccc}
\hline HJD $(+240$ 0000) & Error & Type & Filter \\
\hline 54213.4434 & 0.0014 & I & $B V R$ \\
54219.3903 & 0.0032 & I & $B V R$ \\
54226.3264 & 0.0014 & I & $B V R$ \\
54526.5460 & 0.0008 & I & $B V R$ \\
54587.4837 & 0.0007 & I & $B V R$ \\
54621.4215 & 0.0019 & II & $I$ \\
54651.3956 & 0.0067 & I & BVRI \\
54659.3202 & 0.0038 & I & $B V R I$ \\
54671.4488 & 0.0097 & II & $B V R I$ \\
55675.4011 & 0.0005 & I & $B V R I$ \\
\hline
\end{tabular}

ROTSE $1 V$-band data from the NSVS database (Woźniak et al. 2004) and also the available data in the ASAS database (Pojmánski 1997). In addition, we took the observations published by Bernhard \& Frank (2006) and Frank \& Bernhard (2007). The standard $V$-band data of ROTSE 1 cover the observing seasons of 1999 and 2000, while the standard $V$-band data of ASAS cover the seasons from 2003-2006. The observations in this study were started in 2007. Although the data taken from Bernhard \& Frank (2006) and Frank \& Bernhard (2007) were not standard, using the comparison and check stars given by them we were able to transform to the standard system. After transforming their data, we compared all available data to ascertain whether the data taken from different sources are suitable to use together. To this end, we compared the ASAS data of 2005 and 2006 with the data taken from Bernhard \& Frank (2006) and also compared the data taken from Frank \& Bernhard (2007) with our 2007 data. As seen from the comparisons, the data taken from different sources are statistically the same in $3 \sigma$ value. The light curves obtained from the available data are shown in Figure 2. As seen from the figure, the light variation shapes of seasons 1999, 2000, and 2005 are similar to the variation observed in season 2011. The light curves obtained in 2011 are similar to the light curves obtained in previous studies. Fortunately, I-band observations of 2008 gave us a chance: we can perform light-curve analysis. In the case of a lack of secondary minimum, light-curve analysis does not give reliable results.

\section{Light-Curve Analysis}

The light-curve analysis of such a magnetically active star is generally quite difficult due to the absence of secondary
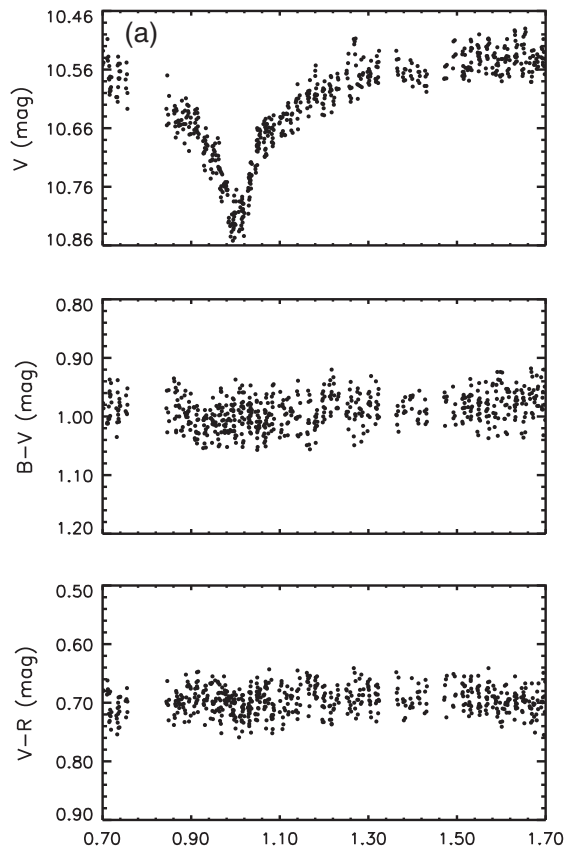
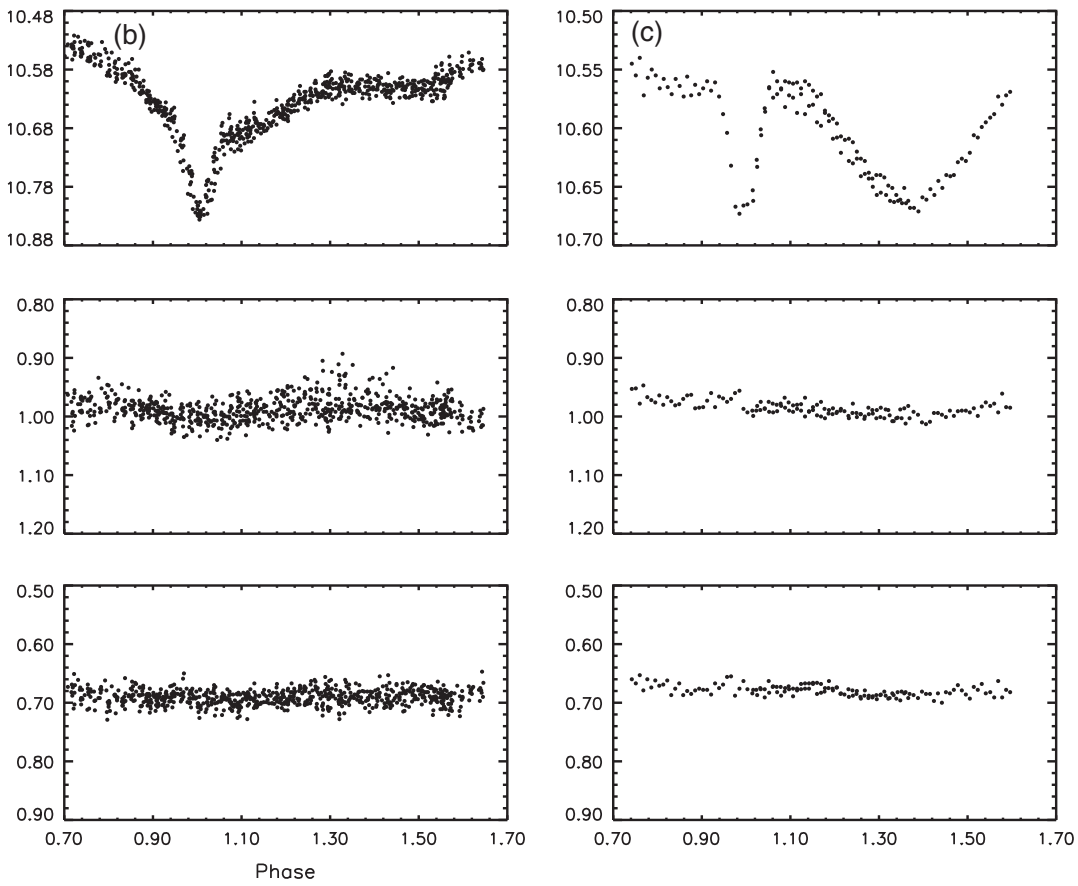

Figure 1 The light and colour curves of GSC 02038-00293 for the observing seasons (a) 2007, (b) 2008, and (c) 2011. 
minima. In fact, no secondary minimum is seen in our observations for the season 2007 and also generally in all other observations published in the literature. However, $I$-band observations in 2008 clearly show the secondary minimum. This gave us the chance to perform light-curve analysis. This is why we analysed only the $I$ light curve obtained in 2008 with the PHOEBE V.0.31a software (Prša \& Zwitter 2005), the method in which depends on the method used in the 2003 version of the WilsonDevinney Code (Wilson \& Devinney 1971; Wilson 1990). We tried to analyse the $I$-band curve with three different modes, the 'detached system', 'semi-detached system with the primary component filling its Roche lobe' and 'semi-detached system with the secondary component filling its Roche lobe' modes. The initial analyses demonstrated that an astrophysically acceptable result can be obtained if the analysis is carried out in the 'detached system' mode. The initial experience revealed that no acceptable results in the astrophysical sense could be obtained in any other mode.

Using low-resolution spectra, Korhonen et al. (2010) found that the system is of $\mathrm{K}$ spectral type, and the $T_{\text {eff }}$ was found to be $4750 \pm 250 \mathrm{~K}$. Considering this case, the temperature of the primary component was fixed to $4750 \mathrm{~K}$ and the temperature of the secondary was taken as a free parameter in the analyses. Considering the spectral type, the albedos $\left(A_{1}\right.$ and $\left.A_{2}\right)$ and gravity-darkening coefficients $\left(g_{1}\right.$ and $\left.g_{2}\right)$ of the components were adopted for stars with convective envelopes (Lucy 1967; Rucinski 1969). The non-linear limb-darkening coefficients $\left(x_{1}\right.$ and $\left.x_{2}\right)$ of the components were taken from van Hamme (1993). In the analyses, the dimensionless potentials $\left(\Omega_{1}\right.$ and $\left.\Omega_{2}\right)$, fractional luminosity $\left(L_{1}\right)$ of the primary component and inclination $(i)$ of the system were taken as adjustable free parameters.

There is no obtained spectroscopic mass ratio for the system. Because of this, we used the 'q-search' method with a step of 0.05 to find the best photometric mass ratio of the components. The general result of the q-search method is shown in Figure 3. As seen from the figure, the minimum sum of weighted squared residuals $\left(\Sigma_{\text {res }}^{2}\right)$ is found for the mass ratio value of $q=0.35$. According to this result, we assume that a possible mass ratio for the system is $q=0.35$.
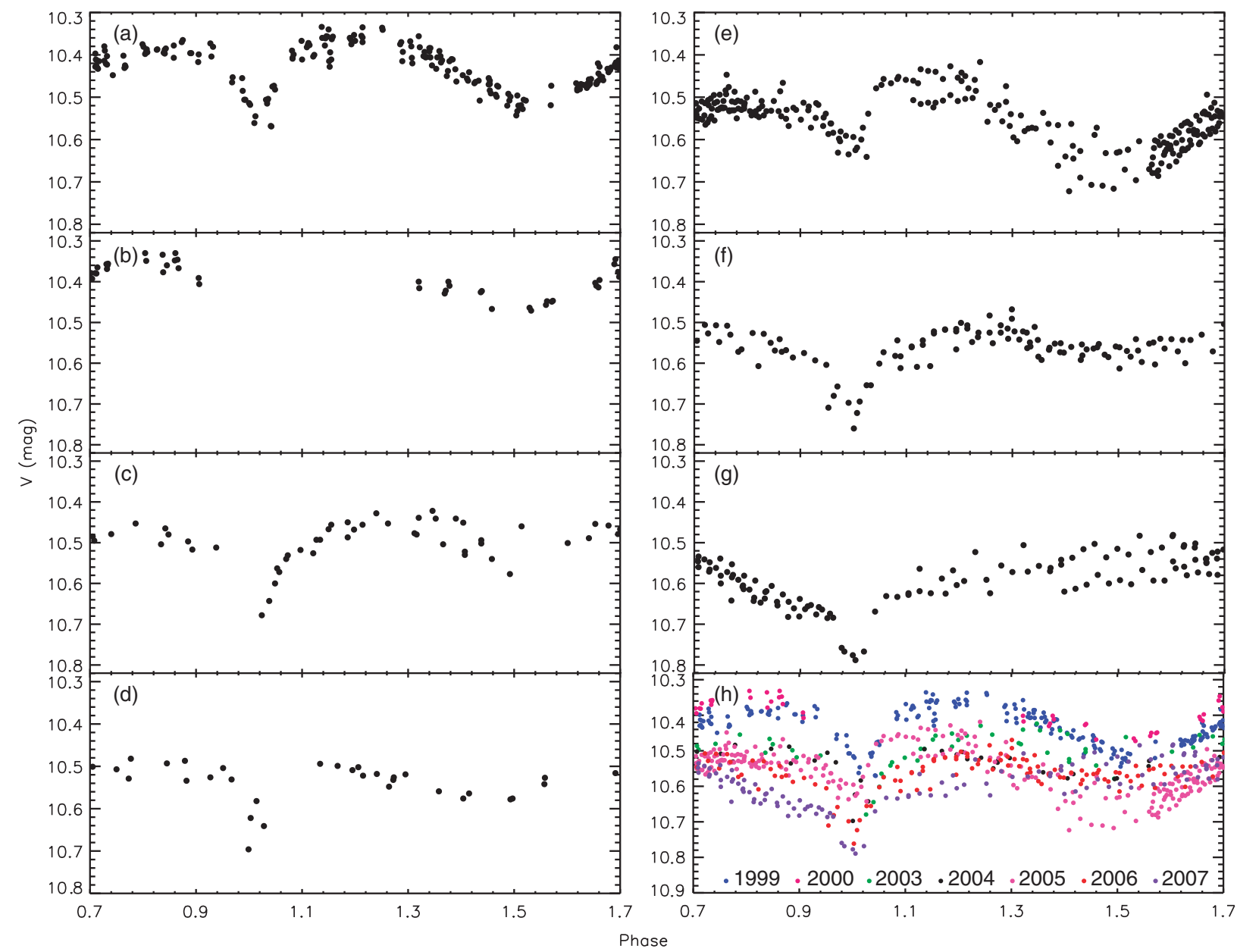

Figure 2 The available observations of GSC 02038-00293 in the literature. In panels (a) and (b), the light curves of the ROTSE $1 V$-band data are shown for the seasons 1999 and 2000. The $V$-band light curves of (c) 2003, (d) 2004, (e) 2005, (f) 2006, and (g) 2007 show data taken from the ASAS database (Pojmánski 1997) and from Bernhard \& Frank (2006) and Frank \& Bernhard (2007). For the seasons 2005 and 2006, there are some data in both the ASAS database and Bernhard \& Frank (2006) and Frank \& Bernhard (2007). For easy comparison, all data sets are shown together in panel (h). 
As is clearly seen from Figures 1 and 2, there is a dramatic asymmetry in the light curves due to the magnetic activity. In the light-curve analysis, we assumed that the primary component has two cool spots on its surface to remove this asymmetry. According to the results obtained from the first iterations, the secondary component seems to be an M dwarf, which is close to the full-convective boundary (Browning 2011). Full-convective M dwarfs exhibit very strong flare activity, while a few of them purely exhibit spot activity (Dal \& Evren 2011). In addition, if the secondary component is the spotted one, to remove the asymmetries seen in the light curves the analysis demonstrated that the spots should be large enough to cover the entire surface of the star or else their effective temperatures must be half of the surface temperature due to the secondary component's light rate in the total light of the system. Although there are a few stars that are close to the full-convective boundary and have some large spots on their surface, this is not a common

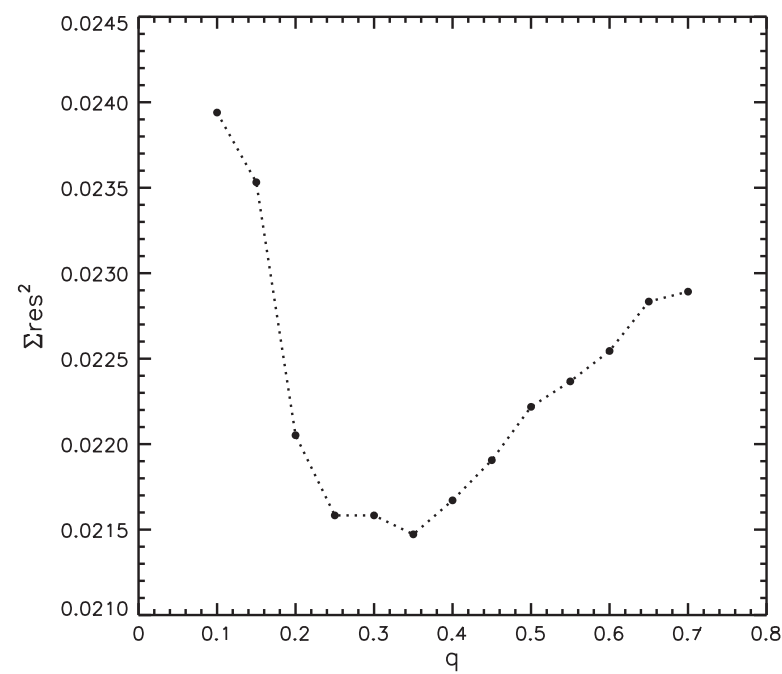

Figure 3 The variation of the sum of weighted squared residuals versus mass ratio in the 'q search'. phenomenon. However, $\mathrm{K}$ dwarfs are generally potential stars, which may possibly exhibit spot activity. This is why we have assumed that the spotted star is the primary component. Moreover, light-curve analyses with this assumption gave more acceptable results in the astrophysical sense. On the other hand, it must be noted here that it is well known that the spot solution suffers from nonuniqueness. The synthetic light curve obtained from the light-curve solution is seen in Figure 4, and the resulting parameters of the analysis are also listed in Table 3.

Although there is not any available radial velocity curve, we tried to estimate the absolute parameters of the components. According to Tokunaga (2000), the mass of the primary component must be $0.73 \pm 0.05 \mathrm{M}_{\odot}$ corresponding to its surface temperature. Considering the possible mass ratio of the system, the mass of the secondary component was found to be $0.25 \pm 0.04 \mathrm{M}_{\odot}$.

Using Kepler's third law, we calculated the possible semi-major axis as a $2.62 \mathrm{R}_{\odot}$. Considering this estimated semi-major axis, the radius of the primary component was computed as $0.87 \pm 0.05 \mathrm{R}_{\odot}$, while that of the secondary component was computed as $0.27 \pm 0.04 \mathrm{R}_{\odot}$. As can be seen from these results, the primary component's radius derived with the assumption of a spotted primary component is in agreement with the value of $R \sin i$ found by Korhonen et al. (2010). Using the estimated radii and obtained temperatures of the components, the luminosity of the primary component was estimated to be $0.35 \mathrm{~L}_{\odot}$ and that of the secondary component was found to be $0.01 \mathrm{~L}_{\odot}$. The absolute parameters are generally acceptable in the astrophysical sense. However, the radius of the primary component is larger than the expected values with respect to the theoretical models. We plotted the distribution of radii versus masses for some stars in Figure 5. The filled circles in the figure represent wellknown active stars listed in the catalogue of Gershberg et al. (1999). Some of these stars exhibit spot activity, while some of them exhibit flare activity. Some stars exhibit both spot and flare activity. In the figure, the asterisk represents the secondary component while the

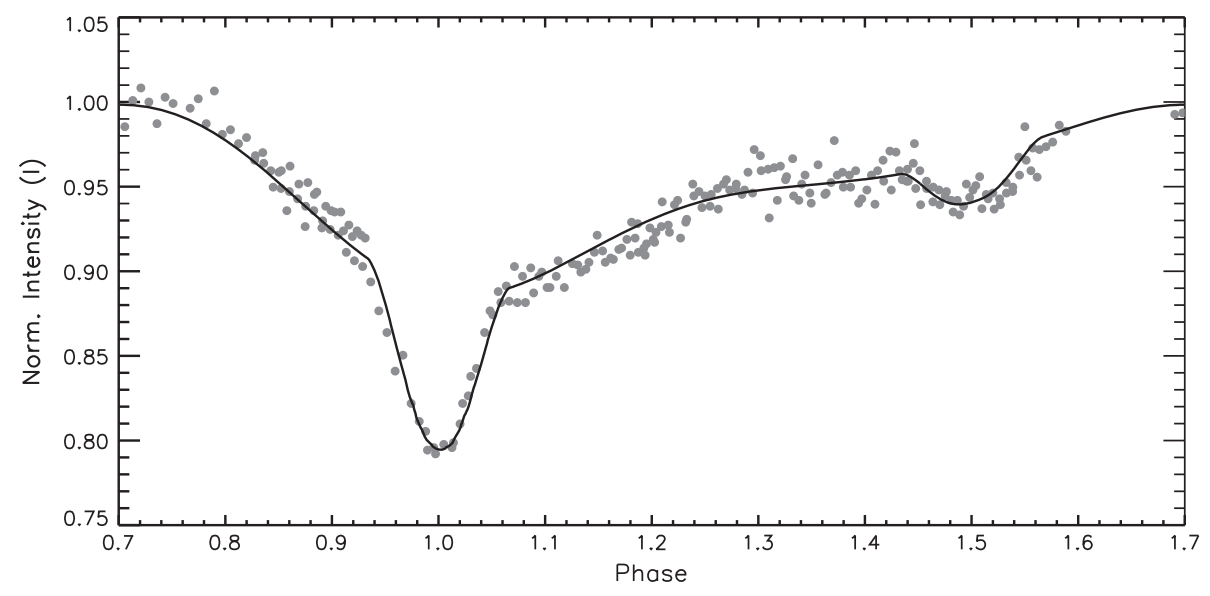

Figure 4 The synthetic light curve obtained from light-curve analysis of the $I$ band. 
open triangle represents the primary component. The line represents the zero-age main sequence (ZAMS) theoretical model developed for stars with $Z=0.02$ by Siess et al. (2000).

Table 3. Parameters obtained from I-band light-curve analysis

\begin{tabular}{ll}
\hline Parameter & Value \\
\hline$q$ & 0.35 \\
$i\left({ }^{\circ}\right)$ & $77.91 \pm 0.87$ \\
$T_{1}(\mathrm{~K})$ & 4750 (Fixed) \\
$T_{2}(\mathrm{~K})$ & $3515 \pm 61$ \\
$\Omega_{1}$ & $3.42 \pm 0.05$ \\
$\Omega_{2}$ & $4.72 \pm 0.14$ \\
$L_{1} / L_{T}(\mathrm{I})$ & $0.977 \pm 0.067$ \\
$g_{1}, g_{2}$ & $0.32,0.32$ \\
$A_{1}, A_{2}$ & $0.5,0.5$ \\
$x_{1, \text { bol }}, x_{2, \text { bol }}$ & $0.625,0.625$ \\
$x_{1, I}, x_{2, I}$ & $0.681,0.681$ \\
$<r_{1}>$ & $0.334 \pm 0.006$ \\
$<r_{2}>$ & $0.103 \pm 0.004$ \\
Co-Lat $_{\text {Spot I }}\left({ }^{\circ}\right)$ & 90.00 (fixed) \\
Long $_{\text {Spot I }}\left({ }^{\circ}\right)$ & 0.00 (fixed) \\
$R_{\text {Spot I }}\left({ }^{\circ}\right)$ & 54.43 (fixed) \\
$T_{\text {eff, Spot I }}$ & 0.97 (fixed) \\
Co-Lat $_{\text {Spot II }}\left({ }^{\circ}\right)$ & 90.00 (fixed) \\
Long $_{\text {Spot II }}\left({ }^{\circ}\right)$ & 263.56 (fixed) \\
$R_{\text {Spot II }}\left({ }^{\circ}\right)$ & 54.43 (fixed) \\
$T_{\text {eff, Spot II }}$ & 0.98 (fixed) \\
\hline &
\end{tabular}

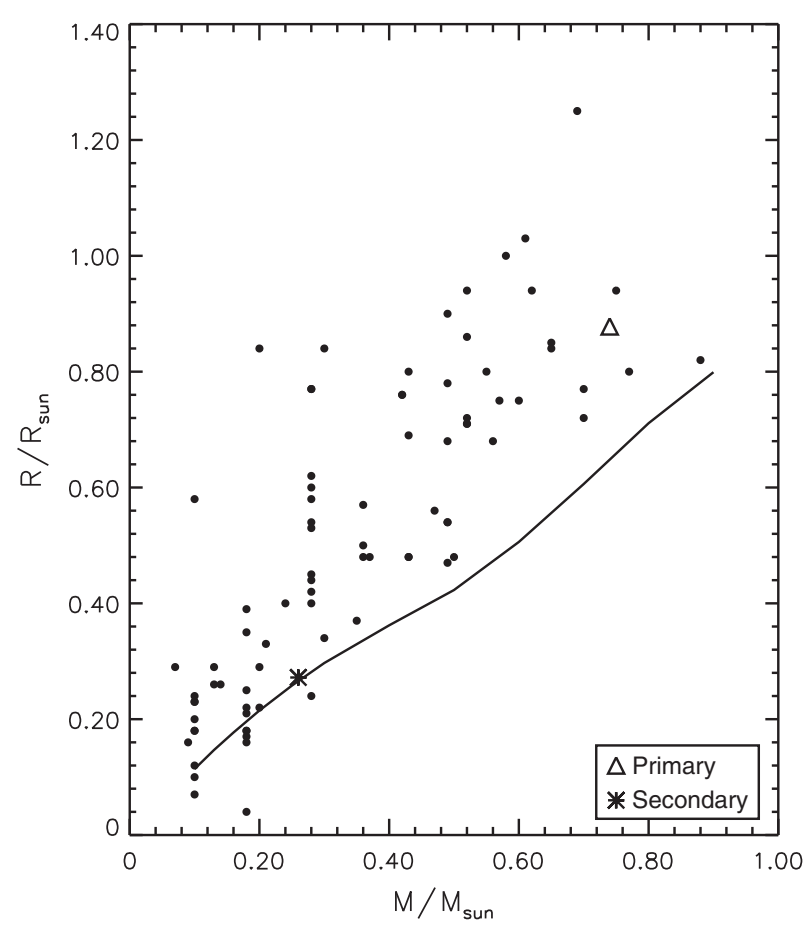

Figure 5 The place of the components of GSC 02038-00293 among well-known active stars in the mass-radius distribution. In the figure, the filled circles represent the active stars listed in the catalogue of Gershberg et al. (1999), the asterisk represents the secondary component, while the open triangle represents the primary component of GSC 02038-00293. The line represents the ZAMS theoretical model developed by Siess et al. (2000).

\section{Variations during Out-of-Eclipses}

The light-curve analysis demonstrated that the light variation of the system is caused by eclipses combined with the effects of magnetic activity existing on the primary component. Considering the contact times derived from the theoretical synthetic light curve, we removed the eclipses from all the $V$-band light curves, and we obtained the remaining curve for each season. Then, in order to reveal the magnetic activity behaviour over the years, we investigated the data during out-of-eclipses firstly for short-term and secondly for long-term variations.

To reveal the spot configuration on the primary component's surface (especially the longitudinal distributions of the spotted areas) in each short-term interval, we modelled the remaining curves under some assumptions using the SPOTMODEL program (Ribárik 2002; Ribárik et al. 2003). Although the data obtained in this study contain multi-band observations, the available data in the literature do not. All data taken from both NSVS and ASAS databases and also from both Bernhard \& Frank (2006) and Frank \& Bernhard (2007) contain only $V$-band observations. However, modelling progress in the SPOTMODEL program requires at least two band observations or any spot temperature factor. Although a large part of the data used is monochromatic, it is likely that we can obtain temperature factors for the spotted areas from light-curve analysis of $I$-band observations during the season 2008 . According to the surface temperature of the primary component, the derived temperature factors are in agreement with the temperature factors found from other analogue stars (Thomas \& Weiss 2008). Considering the spot temperature factor derived from light-curve analysis, we assumed that the temperature factors of the spotted areas are 0.80 in the SPOTMODEL program and the temperature factor was taken as a constant parameter for each model in order simply to determine the surface distributions of the spotted areas for each short-term interval. The longitudes, latitudes, and radii of the spots were taken as adjustable parameters in the program for the model of each season.

The derived models are shown in Figure 6. The models of the data taken from the NSVS database are shown in panels (a) and (b), while the ASAS data are in panels (c) and (d). For seasons 2005 and 2006, there are some data in both the ASAS database and Bernhard \& Frank (2006) and Frank \& Bernhard (2007). These data were combined for each year. The models of these years are shown in panels (e) and (f). For season 2007, some data were obtained in this study and also some data were taken from Frank \& Bernhard (2007). The combined data were modelled, and the result is shown in panel (g). Panels (h) and (i) show models of light curves obtained in seasons 2008 and 2011. The parameters of the models are listed in Table 4 . In the table, the data set, mean times of observations, and mean years of observations are listed in the first three columns, while the spot parameters such as longitude $(I)$, latitude $(b)$, and radius $(g)$ are listed in the following columns for the two spots, respectively. 


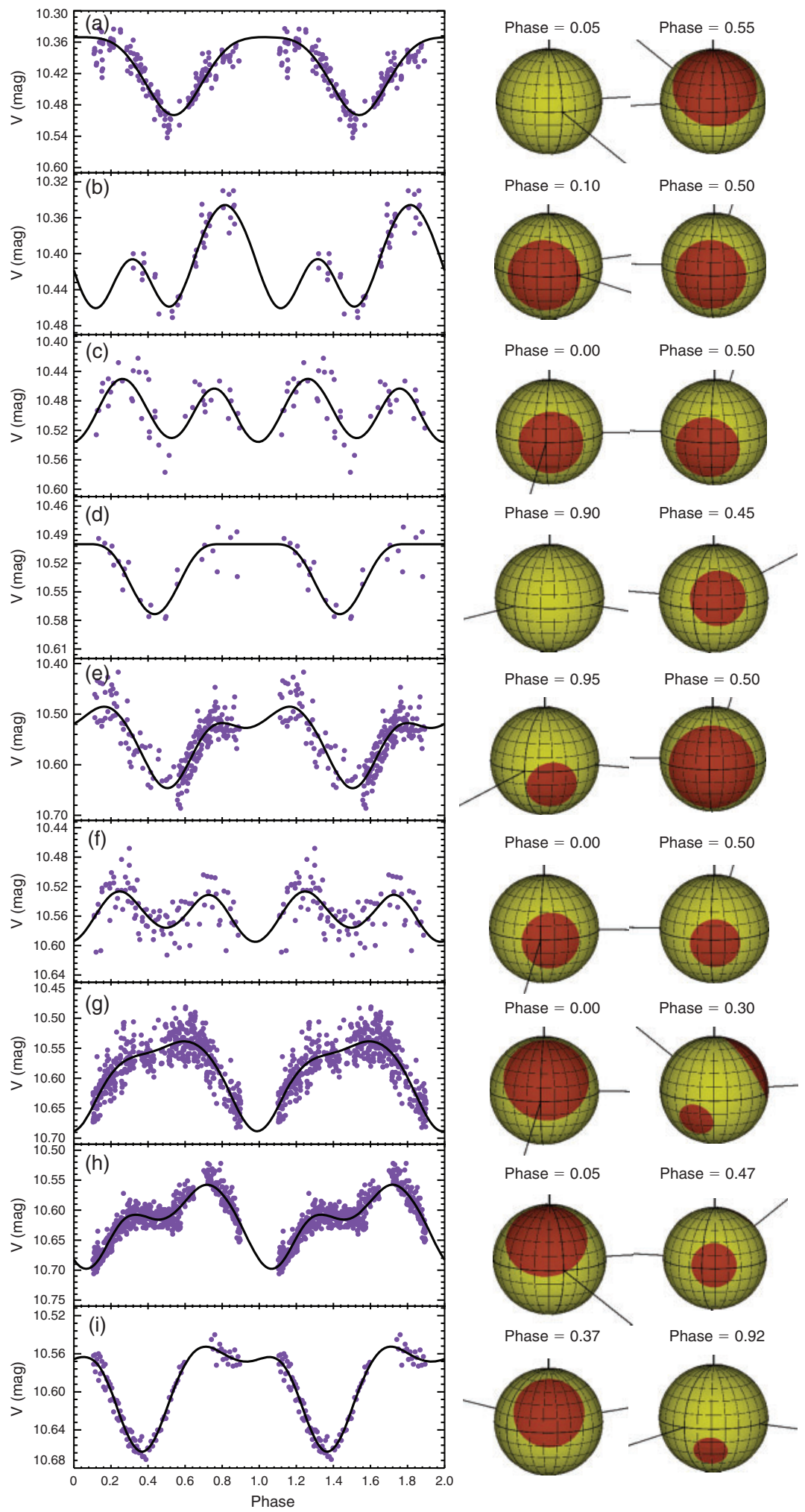

Figure 6 The synthetic light curves during out-of-eclipses and 3D surface models. In each panel on the left side, the filled circles represent the observations while the lines represent the theoretical fit derived by the SPOTMODEL program. The 3D surface model for two phases (especially the phases in which spots are seen) is shown just on the right side of the light curve of each model.

As expected, the spot distribution on the surface is rapidly changing: the distributions vary from one year to the next. As seen from Figure 6, there are usually two spotted areas on the surface of the primary star. Figure 7 demonstrates that the spotted areas are always separated from each other. Although there is usually about $180^{\circ}$ longitudinal difference between them, they sometimes become closer to each other. As seen from Figure 7, the longitude of one of the spotted areas exhibits a quasi-sinusoidal variation over the years, while both of 
Table 4. Spot parameters derived by the SPOTMODEL program for each data set

\begin{tabular}{lccccccccc}
\hline $\begin{array}{l}\text { Data } \\
\text { Set }\end{array}$ & $\begin{array}{c}\text { Mean HJD } \\
(+2450000)\end{array}$ & $\begin{array}{c}\text { Mean } \\
\text { Year }\end{array}$ & $\begin{array}{c}l_{1}^{a} \\
\left({ }^{\circ}\right)\end{array}$ & $\begin{array}{c}l_{2} \\
\left({ }^{\circ}\right)\end{array}$ & $\begin{array}{c}b_{1} \\
\left({ }^{\circ}\right)\end{array}$ & $\begin{array}{c}b_{2} \\
\left({ }^{\circ}\right)\end{array}$ & $\begin{array}{c}g_{1} \\
\left({ }^{\circ}\right)\end{array}$ & $\begin{array}{c}g_{2} \\
\left({ }^{\circ}\right)\end{array}$ & $\begin{array}{c}\text { Data } \\
\text { source }\end{array}$ \\
\hline A & 1350.2176 & 1999.46 & 10 & - & 144 & - & 53 & - & 1 \\
B & 1607.0397 & 2000.17 & 39 & - & 358 & - & 43 & - & 1 \\
B & 1607.0397 & 2000.17 & - & 181 & & 358 & - & 43 & 1 \\
C & 2784.3294 & 2003.39 & 354 & - & 357 & - & 38 & - & 2 \\
C & 2784.3294 & 2003.39 & - & 186 & & 351 & - & 37 & 2 \\
D & 3131.0668 & 2004.34 & 333 & - & 168 & - & 31 & - & 2 \\
E & 3553.9587 & 2005.50 & 330 & - & 342 & - & 28 & - & 2,3 \\
E & 3553.9587 & 2005.50 & - & 178 & & 357 & - & 55 & 2,3 \\
F & 3881.8078 & 2006.39 & 349 & - & 357 & - & 32 & - & 2,3 \\
F & 3881.8078 & 2006.39 & - & 175 & & 355 & - & 28 & 2,3 \\
G & 4255.8110 & 2007.42 & 352 & - & 30 & - & 51 & - & 3,4 \\
G & 4255.8110 & 2007.42 & - & 127 & & 24 & - & 20 & 3,4 \\
H & 4624.8493 & 2008.43 & 20 & - & 40 & - & 47 & - & 4 \\
H & 4624.8493 & 2008.43 & - & 166 & & 3 & - & 26 & 4 \\
I & 5683.9083 & 2011.33 & 331 & - & 332 & - & 18 & - & 4 \\
I & 5683.9083 & 2011.33 & - & 129 & & 24 & - & 41 \\
\hline
\end{tabular}

${ }^{\mathrm{a}}$ In the table, subscripts 1 and 2 represent Spot I and Spot II.

Data sources:

${ }^{1}$ The NSVS database (Woźniak et al. 2004).

${ }^{2}$ The ASAS database (Pojmánski 1997).

${ }^{3}$ Bernhard \& Frank (2006) and Frank \& Bernhard (2007)

${ }^{4}$ This study.

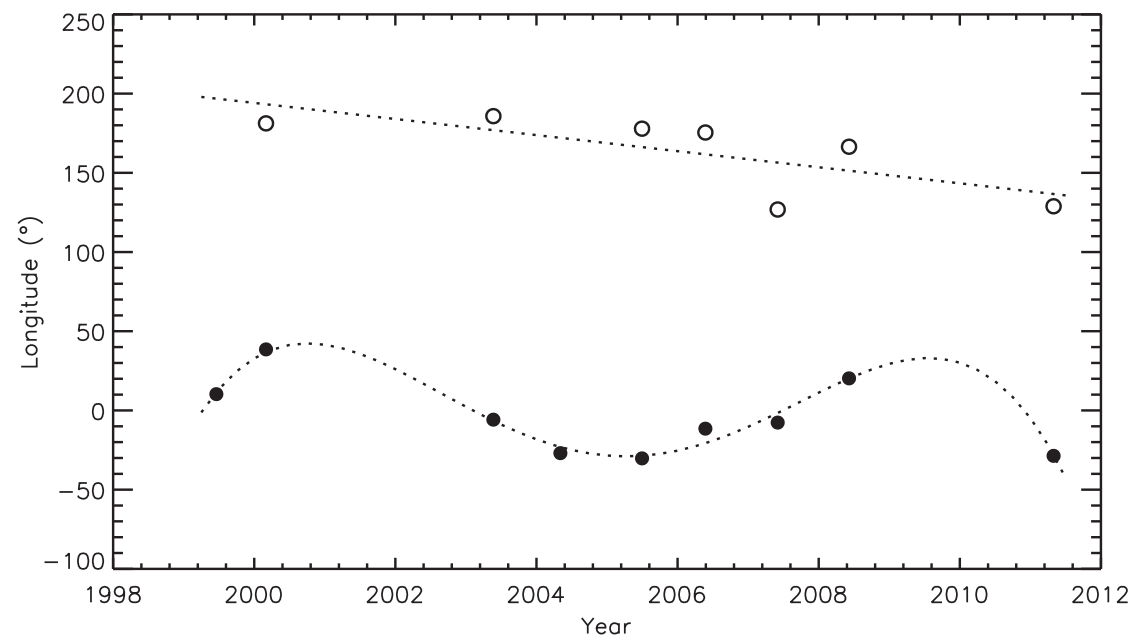

Figure 7 Variation of longitude of the spotted areas. In the figure, filled circles represent Spot I, while the circles represent Spot II. The dashed lines represent the linear fit for Spot I and the polynomial fir for Spot II.

them migrate toward decreasing longitudes. Using GRAPHPADPRISM V5.02 software (Motulsky 2007), the quasi-sinusoidal variation was fitted by a polynomial function. To test whether the polynomial fit is statistically acceptable, we computed the probability value (hereafter $p$-value). The value of $\alpha$ was taken as 0.005 for the $p$-value, which allowed us to test whether the $p$-value are statistically acceptable or not (Dawson \& Trapp 2004). The $p$-value was found to be $<0.00167$. Considering the $\alpha$ value, this means that the result is statistically acceptable.

Apart from the short-term variations, considering the remaining $V$-band light curves without any eclipses we examined the variations of mean brightness, amplitude, and the deeper minimum phases of the light curves $\left(\theta_{\min }\right)$. All of them are listed in Table 5. The variations of three parameters are shown versus the year in Figure 8. The variation of the amplitudes for the remaining curves is shown in panel (a), while the mean brightness variation is shown in panel (b). As is seen from the figures, the amplitude varies in a sinusoidal way with a period of $\sim 8.9$ yr. Using GraphPADPrism V5.02 software, the variation was fitted by a polynomial fit. According to the statistical analysis, just two points, which are shown by open circles in the figure, diverged from the general trend. Apart from these points, the polynomial function fits the 
Table 5. Some parameters determined from the remaining $V$-band light curves

\begin{tabular}{lccccccrr}
\hline $\begin{array}{l}\text { Data } \\
\text { Set }\end{array}$ & $\begin{array}{c}\text { Mean HJD } \\
(+2450000)\end{array}$ & $\begin{array}{c}\text { Mean } \\
\text { Year }\end{array}$ & $\theta_{\min }$ & $\begin{array}{c}V_{\min } \\
(\mathrm{mag})\end{array}$ & $\begin{array}{c}V_{\max } \\
(\mathrm{mag})\end{array}$ & $\begin{array}{c}\text { Amplitude } \\
(\mathrm{mag})\end{array}$ & $\begin{array}{c}V_{\text {mean }} \\
(\mathrm{mag})\end{array}$ & $\begin{array}{c}\text { Data } \\
\text { source }\end{array}$ \\
\hline A & 1350.2176 & 1999.46 & 0.530 & 10.519 & 10.353 & 0.166 & 10.436 & 1 \\
B & 1607.0397 & 2000.17 & 0.520 & 10.457 & 10.340 & 0.117 & 10.399 & 1 \\
C & 2784.3294 & 2003.39 & 0.520 & 10.534 & 10.441 & 0.093 & 10.488 & 2 \\
D & 3131.0668 & 2004.34 & 0.435 & 10.571 & 10.496 & 0.075 & 10.534 & 2 \\
E & 3553.9587 & 2005.50 & 0.495 & 10.648 & 10.479 & 0.169 & 10.564 & 2,3 \\
F & 3881.8078 & 2006.39 & 0.983 & 10.602 & 10.521 & 0.081 & 10.562 & 2,3 \\
G & 4255.8110 & 2007.42 & 0.980 & 10.676 & 10.536 & 0.140 & 10.606 & 3,4 \\
H & 4624.8493 & 2008.43 & 1.050 & 10.702 & 10.539 & 0.163 & 10.621 & 4 \\
I & 5683.9083 & 2011.33 & 0.370 & 10.662 & 10.547 & 0.115 & 10.605 & 4 \\
\hline
\end{tabular}

Data sources:

${ }^{1}$ The NSVS database (Woźniak et al. 2004).

${ }^{2}$ The ASAS database (Pojmánski 1997).

${ }^{3}$ Bernhard \& Frank (2006) and Frank \& Bernhard (2007).

${ }^{4}$ This study.

general trend with a correlation coefficient of 0.91 . Moreover, the $p$-value was found to be $<0.00093$. Considering the $\alpha$ value and $p$-value, the result is statistically acceptable. However, the mean brightness is dramatically decreasing through the years 1999-2011. During these years, it decreases from 10.40-10.60 mag. Both figures indicate that the primary star has a high level of magnetic activity. The distribution of the deeper minimum phases of the remaining curves is shown in the bottom panel of Figure 8.

As seen from Figure 8, the deeper minimum was located in the phase interval between 0.35 and $0.55 P$ from 1999-2005. However, it was suddenly shifted to the phase interval between 0.95 and $0.05 P$ from 2006-2008. It was again seen in the phases between 0.35 and $0.355 \mathrm{P}$ in season 2011. The phenomenon is generally referred to as 'flip-flop' in the literature (Korhonen \& Elstner 2005; Berdyugina 2006; Oláh et al. 2006; Korhonen \& Järvinen 2007). Considering the minima seen in the phase interval between 0.35 and $0.55 P$ reveals that the minima slowly migrate toward decreasing phases.

\section{Results and Discussion}

In this study, we have tried to reveal the nature of the close binary system GSC 02038-00293. Light-curve analysis indicated that the mass ratio of the system is 0.35 . We estimated that the mean radii are $0.87 \mathrm{R}_{\odot}$ for the primary and $0.27 \mathrm{R}_{\odot}$ for the secondary component. Korhonen et al. (2010) indicated that the effective temperature is $4750 \mathrm{~K}$ and found $R \sin i=0.88 \mathrm{R}_{\odot}$. In this study, the inclination $(i)$ of the system was found to be $77.91^{\circ}$. In this case, the radius of the primary component estimated in this study is in agreement with the one given by Korhonen et al. (2010). On the other hand, the radius of the primary star is actually larger than the expected value. As is seen from Figure 5, the secondary component is located almost on the ZAMS. This indicates that this component should be very young. However, it seems that the primary component has departed from the ZAMS. The most likely
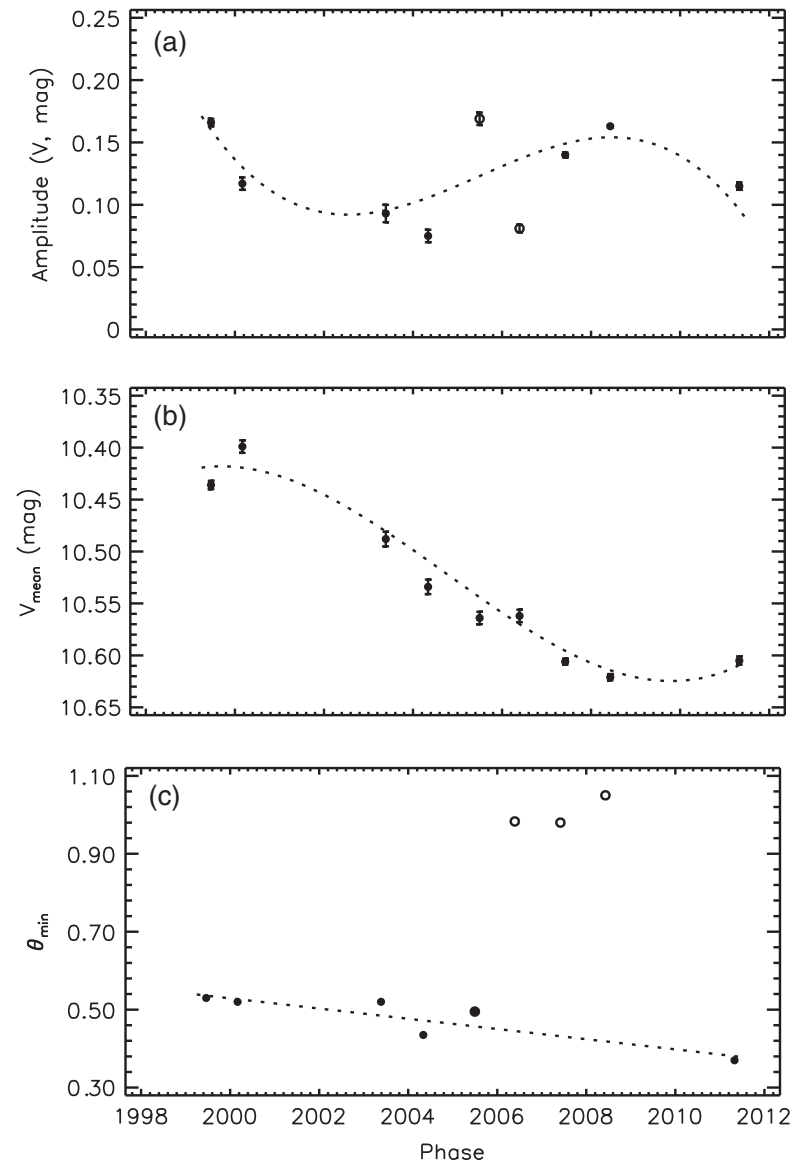

Figure 8 Variation of some parameters determined from the remaining curves: (a) amplitude variation, (b) mean brightness variation, and (c) spot minimum phase distribution. The filled circles represent the parameters determined in the figure, while the dashed lines represent polynomial fits in panels (a) and (b), and the linear fit for $\theta_{\min }$ of one spotted area. The open circles in panel (a) represent points that diverge from the general trend.

situation is that, considering their masses, the primary component has evolved more rapidly than the secondary component. On the other hand, as was discussed by López-Morales (2007), Morales et al. (2008, 2010), 
Casagrande et al. (2008), Fernandez et al. (2009), Torres et al. (2010) and Kraus et al. (2011), the case of larger radius is a common phenomenon for active stars. LópezMorales (2007) demonstrated that this case is very common, especially for magnetically active stars in the mass range $0.35-0.70 \mathrm{M}_{\odot}$. The mass of the primary star of the system is close to this interval. Consequently, it is possible that its radius was found to be larger due to magnetic activity. The radius of the secondary component is in agreement with the expected value for a star with mass $0.25 \mathrm{M}_{\odot}$.

Light-curve analysis of I-band observations of season 2008 and spot models of the light curves during out-of-eclipses confirmed that there are two spotted areas on this component. The models and examination of variations during out-of-eclipses generally reveal some properties as regards the primary component's magnetic activity behaviour. (1) There are two active longitudes on the primary component. (2) One of them is usually active, while the second one can sometimes be less active. (3) Although there are two stable active longitudes on the star, the locations of the spotted areas on this star can change rapidly. Both active longitudes migrate toward decreasing longitude. In addition, one of them exhibits a quasi-sinusoidal variation during the migration. Here, it should be noted that we have assumed that the temperature factors of the spots are stable and constant over the years. Because of this assumption, the radii and latitudes of the spotted areas obtained from spot models can clearly change when another temperature factor is taken for the spots. However, the longitudes will not change. The temperature factor, radius, and latitude depend on each other, but the longitude does not depend directly on them.

Examinations of long-term amplitude variation of the light curves during out-of-eclipses indicate that the amplitude is varying in a sinusoidal way, while the mean brightness of the system is dramatically decreasing. In this case, it is possible that the spots should cover a greater part of the surface of the active component, while the spotted areas sometimes gather toward an active longitude. On the other hand, it is also possible that there could be another scenario. The decrease of mean brightness could be due to any spotted polar-cap areas. The increasing total area covered by spots on the polar can cause the same effect on the mean brightness. However, the phases of the deeper minima during out-of-eclipses migrate toward decreasing phase. The other point is that the second active longitude is more active than the first one between seasons 2006 and 2008. This is a small clue to 'flipflop' behaviour, which is a general property seen in many active stars (Berdyugina 2006; Oláh et al. 2006; Korhonen \& Elstner 2005; Korhonen \& Järvinen 2007).

In this study, the nature of the system is made a little clearer. As a result of the analyses, we can say that GSC 02038-00293 is a close binary, the primary component of which exhibits high-level magnetic activity. Long-term photometric observations, together with spectral studies, will reveal the object's nature more fully.

\section{Acknowledgments}

The authors acknowledge generous allotments of observing time at the Ege University Observatory (EUO). Thanks are due to Dr Bernhard, who shared all his data with us, and gave us all the ROTSE 1 and ASAS 3 data of GSC 02038-00293. We also thank the referee for useful comments that have contributed to the improvement of the paper.

\section{References}

Berdyugina, S. V., 2006, IAUJD, 8E, 64

Bernhard, K. \& Frank, P., 2006, IBVS, 5719, 1

Bopp, B. W. \& Evans, D. S., 1973, MNRAS, 164, 343

Bopp, B. W. \& Fekel, F., Jr, 1977, AJ, 82, 490

Browning, M. K., 2011, in IAU Symp. Vol. 271, Astrophysical Dynamics: From Stars to Galaxies, ed. N. H. Brummell, A. S. Brun, M. S. Miesch \& Y. Ponty (Cambridge: CUP), 69

Casagrande, L., Flynn, C. \& Bessell, M., 2008, MNRAS, 389, 585 Dal, H. A. \& Evren, S., 2011, PASJ, 63, 427

Dawson, B. \& Trapp, R. G., 2004, Basic and Clinical Biostatistics (New York: McGraw-Hill), 61

Dragomir, D., Roy, P. \& Rutledge, R. E., 2007, AJ, 133, 2495

Fernandez, J. M., et al., 2009, ApJ, 701, 764

Frank, P. \& Bernhard, K., 2007, OEJV, 71, 1

Friedemann, C. \& Gürtler, J., 1975, AN, 296, 125

Gershberg, R. E., Katsova, M. M., Lovkaya, M. N., Terebizh, A. V. \& Shakhovskaya, N. I., 1999, A\&AS, 139, 555

Hardie R. H., 1962, in Astronomical Techniques, ed. W. A. Hiltner (Chicago: Univ. Chicago Press), 178

Korhonen, H. \& Elstner, D., 2005, A\&A, 440, 1161

Korhonen, H. \& Järvinen, S. P., 2007, in IAU Symp. Vol. 240, Binary Stars as Critical Tools \& Tests in Contemporary Astrophysics, ed. W. I. Hartkopf, E. F. Guinan \& P. Harmanec (Cambridge: CUP), 453

Korhonen, H., Vida, K., Husarik, M., Mahajan, S., Szczygiel, D. \& Oláh, K., 2010, AN, 331, 772

Kraus, A. L., Tucker, R. A., Thompson, M. I., Craine, E. R. \& Hillenbrand, L. A., 2011, ApJ, 728, 48

Kron, G. E., 1952, ApJ, 115, 301

Kunkel, W. E., 1975, in IAU Symp. Vol. 67, Variable Stars and Stellar Evolution, ed. V. E. Sherwood \& L. Plaut (Dordrecht: Reidel), 15

Landolt, A. U., 1983, AJ, 88, 439

Landolt, A. U., 1992, AJ, 104, 340

López-Morales, M., 2007, ApJ, 660, 732

Lucy, L. B., 1967, ZA, 65, 89

Morales, J. C., Ribas, I. \& Jordi, C., 2008, A\&A, 478, 507

Morales, J. C., Gallardo, J., Ribas, I., Jordi, C., Baraffe, I. \& Chabrier, G., 2010, ApJ, 718, 502

Motulsky, H., 2007, GraphPad Prism 5: Statistics Guide (San Diego: GraphPad Software), 94

Norton, A. J., et al., 2007, A\&A, 467, 785

Oláh, K., Korhonen, H., Kövári, Zs., Forgács-Dajka, E. \& Strassmeier, K. G., 2006, A\&A, 452, 303

Pojmánski, G., 1997, AcA, 47, 467

Prša, A. \& Zwitter, T., 2005, ApJ, 628, 426

Ribárik, G., 2002, Occasional Technical Notes from Konkoly Observatory No. 12, available at http://www. konkoly. hu/staff/ribarik/SML/

Ribárik, G., Oláh, K. \& Strassmeier, K. G., 2003, AN, 324, 202

Rodonó, M., 1986, NASSP, 492, 409

Rucinski, S. M., 1969, AcA, 19, 245

Siess, L., Dufour, E. \& Forestini, M., 2000, A\&A, 358, 593 
Sipahi, E., Dal, H. A. \& Özdarcan, O., 2009, IBVS, 5904

Thomas, J. H. \& Weiss, N. O., 2008, in Sunspots and Starspots, Cambridge Astrophysics Series 46, 1st edition, ed. A. King, D. Lin, S. Maran \& J. M. Ward (New York: CUP), 181

Tokunaga, A. T., 2000, in Allen's Astrophysical Quantities, 4th edition, ed. A. N. Cox (Berlin: Springer), 143

Torres, C. A. O. \& Ferraz Mello, S., 1973, A\&A, 27, 231
Torres, G., Andersen, J. \& Giménez, A., 2010, A\&ARv, 18, 67 van Hamme, W., 1993, AJ, 106, 2096

Voges, W., et al., 1999, A\&A, 349, 389

Vogt, S. S., 1975, ApJ, 199, 418

Wilson, R. E., 1990, ApJ, 356, 613

Wilson, R. E. \& Devinney, E. J., 1971, ApJ, 166, 605

Woźniak, P. R., et al., 2004, AJ, 127, 2436 